\title{
Hydrogen peroxide disproportionation: time-resolved optical measurements of spectra, scattering and imaging combined with correlation analysis and simulations
}

\author{
Mohamed E. A. Ibrahim and René A. Nome* \\ Institute of Chemistry, University of Campinas, Brazil, 13083-970. \\ E-mail: nome@unicamp.br
}

\begin{abstract}
We study how time-dependent optical measurements of spectra, scattering, and imaging can be used to add to the understanding of heterogeneous reactions, compared to work performed using tools developed for homogeneous reactions. Using hydrogen peroxide disproportionation by potassium permanganate as a model reaction, we measure the entire spectrum over reaction time, enabling a clear and useful correlation analysis and assignment of chemical species in heterogeneous conditions. We measure time-dependent dynamic light scattering to study oxygen nanobubble product formation kinetics and equilibrium. We perform macroscopic video-rate reaction imaging and information-theoretic analysis to characterize reaction and transport contributions to the observed signal. To illustrate the differences arising from measuring sample subsets vs the entire system, we integrate stochastic and macroscopic numerical simulations of reaction-diffusion to study homogeneous and heterogeneous reaction conditions. We hope the tools presented here may help understanding other chemical reactions in heterogeneous conditions.
\end{abstract}

\section{INTRODUCTION}

Chemical kinetics and laser spectroscopy are powerful tools to study reaction mechanisms. By following reactant and/or product concentrations as function of time over the course of a reaction, a systematic chemical kinetics study enables improved understanding of the underlying energy landscape. Nowadays, with the advent of new technologies that allow measurements at higher sampling rates, larger volumes, and broader spectral range, it is interesting to assess how such tools can be applied in investigations of important yet complex, multiscale reaction mechanisms.[1-5] For example, one of the challenges in trying to understand and improve chemical reactions that use visible sunlight to produce renewable products and solar fuels is the development of new experimental approaches for studying reaction mechanisms occurring on multiple length scales - from molecular to macroscopic - and time scales - from earliest molecular events to real time.[6-9]

Previously, we have studied different ways in which time-resolved laser spectroscopy can be used to learn more about physical chemical processes and reaction mechanisms.[10-12] In ref 12 , using as examples stochastic dynamics simulations of Brownian motion in a bistable asymmetric well, photochemical kinetics of conjugated polymer photo-degradation from experimental solution-filled optical fiber time-resolved fluorescence spectra, and ultrafast spectroscopy for the same system, we discussed how more information about the reaction can be obtained as a function of sampling rate, number of molecules being probed, and spectral range to be studied, respectively. More recently, we have reported mechanistic studies of photocatalytic degradation of organic molecules with in situ micro-spectroscopy and optical tweezers: we monitored images and time-dependent spectra, and quantitative optical microscopy 
was used to characterize spatially-dependent reaction rates and correlate with stochastic dynamics of individual catalytic nanoparticles.[13]

In the present work, we study mechanisms of a model reaction for oxygen evolution with timeresolved spectroscopies, imaging and simulations. We choose to study hydrogen peroxide disproportionation during hydrogen peroxide reaction with potassium permanganate, a well-known reaction.[14-16] It exhibits features (chemical reaction, bubble and particle aggregate formation and growth, mixing, drift, and diffusion effects) which may be relevant for related reactions important in energy applications and artificial photosynthesis. We measured real-time time-dependent extinction spectra to simultaneously monitor reactant and products for a series of reaction conditions. We used independent measurements of absorbance and dynamic light scattering as a function of time to isolate separate response contributions to the observed time-dependent extinction spectra. We recorded transmitted light intensity over the entire sample during the chemical reaction at the macroscopic level and performed an information-theoretic quantitative analysis to study spatially-dependent features of the reaction mechanism. We compared the results coupled stochastic/macroscopic simulations of diffusion and chemical kinetics to understand the applicability of the approach presented herein. Overall, the experiments, analysis and simulations presented here may be generally applied to the study of reaction mechanisms under conditions of varying length and time scales.

\section{EXPERIMENTAL SECTION}

All reagents used were analytical grade. Hydrogen peroxide concentration values used in this work ranged from 0.23 to $0.52 \mu \mathrm{mol} . \mathrm{L}^{-1}$, with permanganate in excess with a 200:1 ratio. The optical spectroscopy measurements were performed by uv-vis-NIR spectroscopy and dynamic light scattering. Briefly, in the uv-vis-NIR measurements, we used a home-built setup including a broadband excitation light source, optics and detection with a camera and spectrograph. This setup enables simultaneous monitoring of transmitted light intensity as a function of wavelength and time, and using that information to calculate extinction. Data analysis algorithms were developed in-house and are based in our previous published works. Further details of the experimental setup and data analysis are described in references [10-13].

\section{QUANTITATIVE REACTION IMAGING}

The spectral assignments described in Figures 1 and 2 were used to identify which component of the measured image (that is: $R, G$, or $B$ ) was associated with each species. Thus, the video images recorded for the entire reaction were separated into three sets: $R, G$, and $B$. The $B$ video data set was assigned to the product species, while the $G$ video data set was assigned to the reactant species. The $R$ video data set was associated with light scattering. For each movie frame, we transform the R, G, and B intensities into an 8-bit scale and assume a linear relationship between 8-bit scale value and the incident light intensity, for every pixel in the image. The resulting intensities were then used to calculate absorbance per pixel and for every frame. We note that the above-described procedure is commonly used to connect pixel intensities to absorbance changes in spectroscopic applications of image processing software.

To further understand the absorbance changes during the reaction performed under the set of initial concentrations where macroscopic bubbles are absent in solution, we performed full sample (that is, the entire cuvette) reaction monitoring with video-rate imaging. Based on the assignments from Figures 1 and 2, the recorded chemical reaction image sequences are split into red, green and blue contributions, which are assigned to scattering, reactant, and product, respectively. For each recorded reaction, we used 
an information-theoretic measure to calculate compositional information from image partitioning at each frame, and plot the information gained at each partitioning and at each time step over the course of the reaction. To achieve this goal, we used the line-updating bi-partitioning (LUB) algorithm to calculate the Shannon entropy $H(C)$, conditional entropy $H(C \mid R)$, and mutual information $I(C, R):[17-20]$

$$
\begin{gathered}
H(C)=-\sum_{c \in C} P(c) \log _{2} P(c) ; P(c)=S_{c} / S \\
H(C \mid R)=-\sum_{c \in C, r \in R} P(c, r) \log _{2} P(c \mid r) ; \\
P(c, r)=\operatorname{Pr}[C=c, R=r] \\
P(c \mid r)=\operatorname{Pr}[C=c \mid R=r] \\
I(C, R)=H(C)-H(C \mid R)
\end{gathered}
$$

where $R$ is the image partitioning into specific regions $(r 1, r 2, \ldots), S c$ is the number of pixels taking color $c$ $(R, G, B)$, and $S$ the image size. We considered vertical and horizontal partitioning of the recorded video of the chemical reaction and calculate the mutual information $I(C, R)$ as a function of time for each frame to determine the maximum information gain.

\section{SIMULATIONS}

To understand the connection between our spectroscopic observables and the chemical reaction, we performed numerical studies of macroscopic reaction-transport coupled with stochastic description of chemical kinetics and diffusion.[21,22] Thus, we look at dynamics of individual particles using the Langevin equation, and we look at the hydrodynamic equations for diffusion in the presence of a chemical reaction of the type $A+B \rightarrow C$. To implement the coupled stochastic-macroscopic reaction-diffusion simulation, our starting point is the macroscopic approach, using the hyperbolic reaction-diffusion equation:[23]

$$
\begin{aligned}
& \tau_{i} \frac{\partial^{2} \rho_{i}}{\partial t^{2}}+\frac{\partial \rho_{i}}{\partial t}=D_{i} \frac{\partial^{2} \rho_{i}}{\partial x^{2}}+F\left(\rho_{i}\right) \\
& \frac{\partial \rho_{i}}{\partial t}=F\left(\rho_{i}\right)
\end{aligned}
$$

where $\rho$ is the concentration of each reactive diffusing species, $D$ is the corresponding diffusion coefficient, and $F(\rho)$ is the reaction rate law, which satisfies the condition of preserving positivity.

To implement the stochastic part, the ad hoc $\tau \partial_{\mathrm{tt}} \rho$ term is replaced in two steps. First, for each particle in the simulation region, the Langevin equation is solved including the inertial term:

$$
m \frac{d^{2} x}{d t^{2}}=-\gamma \frac{d x}{d t}+\sqrt{2 D} \xi(t)
$$

where $\mathrm{m}$ is the particle mass, $\mathrm{x}$ is the particle position, $\gamma$ the friction coefficient, and $\sqrt{2 D} \xi(t)$ is the fluctuating force due to collisions with the solvent molecules. We performed simulations with reflective 
boundary conditions to account for the finite sample volume (sample holder) and without (periodic boundary conditions).[13,24,25]

Second, the macroscopic simulation system is then divided into mesoscopic cells that are much larger than the reactive Brownian particles yet much smaller than the system size. For each cell, we count particle numbers and then determine chemical species concentrations. This information is used as an input for the time-evolution of the system based on the hydrodynamic equations for diffusion and kinetics based on the above chemical equation. In addition to the Langevin dynamics parameters, we also specify reaction rate constants and diffusion coefficients. Therefore, to study the effect of reaction-transport parameters on the spectroscopic observables, we consider how the relative values of diffusion constant vs reaction rate constant influence the numerically calculated rate constants. This analysis is performed by monitoring a portion of the entire simulation system which we denote as "spectroscopic observation window". We then compare how the retrieved information depends on the size of this observation window, all the way up the full system size. The simulations shown below were performed in one dimension with periodic boundary conditions, and we reached similar conclusions for two-dimensional simulations and closed geometry.

\section{RESULTS AND DISCUSSION}

\section{Time-resolved extinction spectroscopy}

(A)

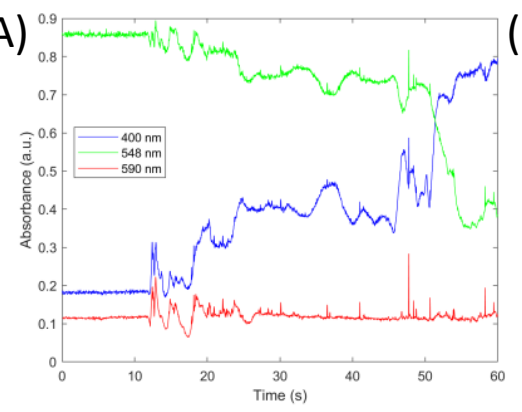

(B),

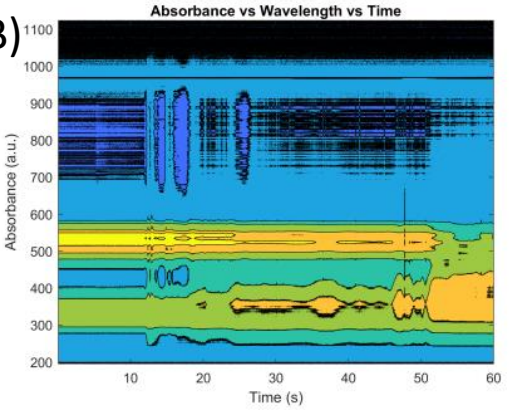

(C)

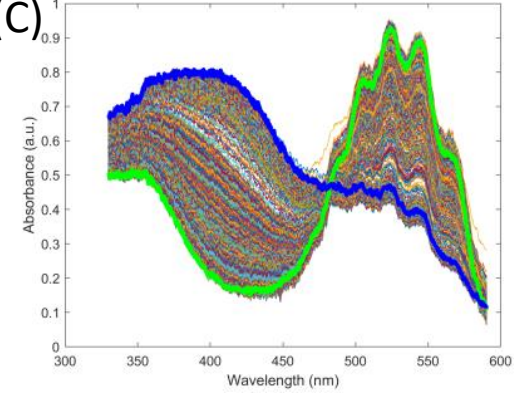

Figure 1. Time-dependent spectra for hydrogen peroxide disproportionation in the presence of potassium permanganate: (A) reaction monitoring at individual wavelengths (taken from Figure 1B): $400 \mathrm{~nm}$ (blue line), $548 \mathrm{~nm}$ (green line) and $590 \mathrm{~nm}$ (red line); (B) contour plot of absorbance vs wavelength vs time; (C) absorbance spectra as a function of time.

Figures $1 \mathrm{~A}, 1 \mathrm{~B}$ and $1 \mathrm{C}$ show our results for real-time time-dependent transmission spectra during the reaction between hydrogen peroxide and potassium permanganate for $1 \mathrm{mmol} . \mathrm{L}^{-1}$ hydrogen peroxide concentration and excess permanganate (see Experimental Section and the Supporting Information). Figure $1 \mathrm{~A}$ illustrates the time evolution of absorbance at three different wavelength values (taken from Figure 1B): $400 \mathrm{~nm}, 538 \mathrm{~nm}$ and $590 \mathrm{~nm}$, corresponding to reactant (permanganate), product (manganese dioxide), and scattering, respectively (compare with Figure S1). In the green curve in Figure $1 \mathrm{~A}$, a net decrease in the absorbance signal at $538 \mathrm{~nm}$ as a function of reaction time can be inferred, which is expected for reactant consumption. Nonetheless, the absorbance profile at $538 \mathrm{~nm}$ also exhibits large fluctuations and nonmonotonic decay over the course of the reaction, and the measured transmitted light intensity reflects a sum of absorption and scattering contributions to the observed total extinction signal. Therefore, although this is a well-known reaction, its mechanistic study highlights the difficulty of using experimental tools of chemical kinetics when the reaction involves bubble formation, phase changes, particle aggregation and growth, transport, and drift, among other processes (see Movie S1). We choose this reaction for further 
investigation precisely because the methodology developed herein to study this reaction can be applied to study other reactions involved in new energy applications, dense energy carriers, and solar fuels.

Figure 1B shows extinction ( $\mathrm{z}$-axis) as a function of wavelength ( $\mathrm{y}$-axis) and as a function of time ( $\mathrm{x}$ axis). Thus, in Figure $1 \mathrm{~B}$ we monitor the chemical reaction at multiple wavelengths simultaneously. Figure $1 \mathrm{C}$ shows the same data as in Figure $1 \mathrm{~B}$, now plotted as spectra taken at successive time intervals. Given the high sampling rate used, in Figure $1 \mathrm{~B}$ we can observe a continuous variation in extinction as a function of time over the entire course of the reaction. Furthermore, as shown in Figure $1 \mathrm{C}$, we can more clearly identify spectral ranges where the extinction decreases as a function of reaction time, which is associated with permanganate reactant consumption. Moreover, at lower wavelengths, increasing extinction is observed, which correlates with increasing manganese dioxide product concentration. An isosbestic-like point can be observed near $485 \mathrm{~nm}$, thus further indicating interconversion between two species in the corresponding spectral ranges centered at $400 \mathrm{~nm}$ and $548 \mathrm{~nm}$. We note, however, that the time-evolution at individual wavelengths is non-monotonic, as exemplified by the traces shown in Figure 1A. Therefore, we look for correlations across the spectrum to characterize the species observed spectroscopically.
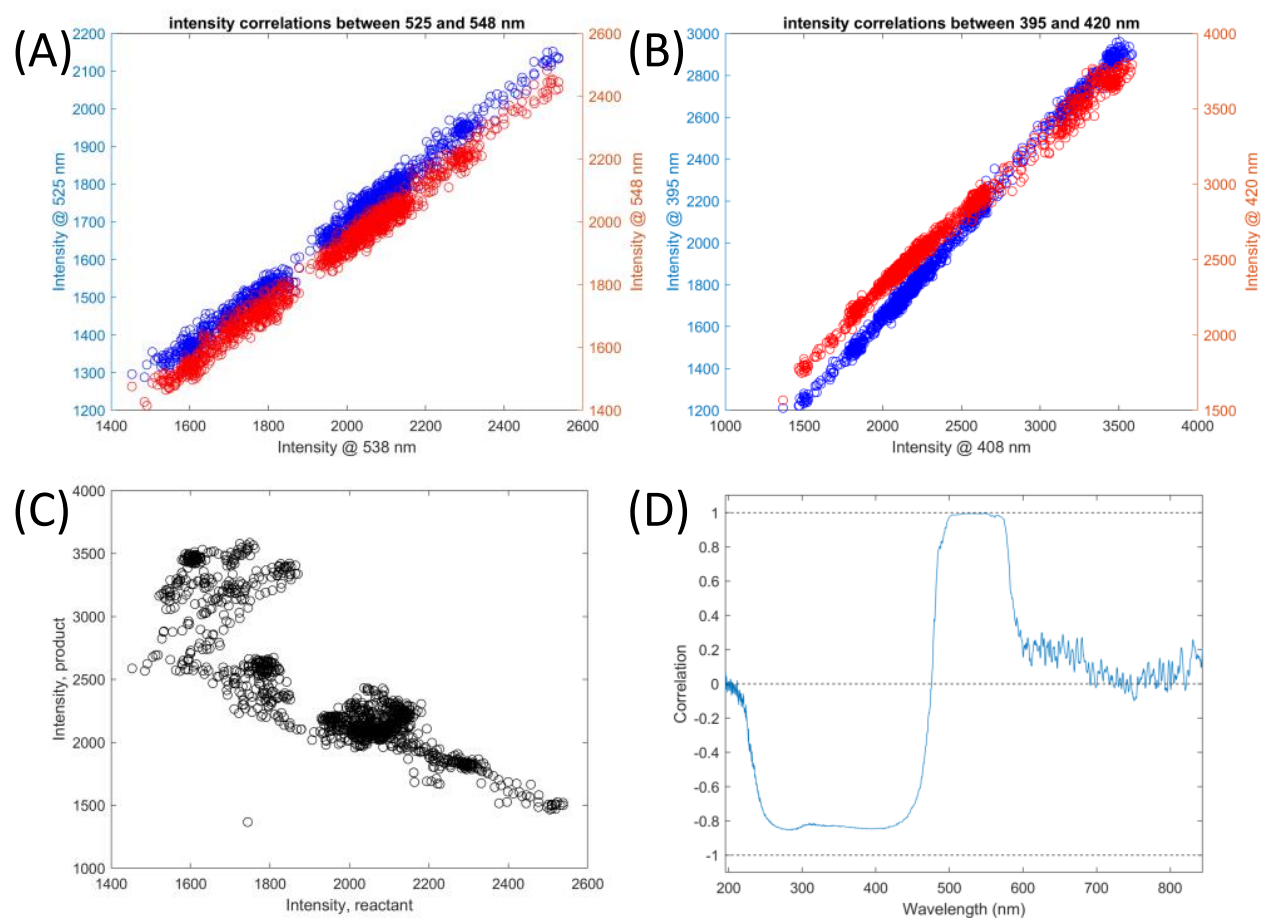

Figure 2. Correlation analysis of time-dependent spectra for hydrogen peroxide disproportionation in the presence of potassium permanganate, using $538 \mathrm{~nm}$ as reference wavelength: $(A)$ intensity correlations between 525 $\mathrm{nm}$ and $548 \mathrm{~nm}$; (B) intensity correlations between $395 \mathrm{~nm}$ and $420 \mathrm{~nm}$; (C) intensity anti-correlations between reactant and product species; and (D) wavelength-dependent correlation averaged over the duration of the reaction.

Figure 2 shows correlation analysis of intensity as a function of time for different spectral ranges associated with reactant and product, as identified in Figure 1C.[17] In Figure 2A, using the time-dependent transmitted light intensity at $538 \mathrm{~nm}$ as a reference, we plot the corresponding time-dependent intensities at $525 \mathrm{~nm}$ and $548 \mathrm{~nm}$ (blue and red points, respectively). Thus, Figure $2 \mathrm{~A}$ shows positive transmitted light intensity correlations over the course of the reaction and across the spectral band assigned to reactant absorption in Figure 1C. Likewise, over the entire reaction time, Figure 2B shows positive transmitted light intensity correlations in the spectral range between $395 \mathrm{~nm}$ and $420 \mathrm{~nm}$, using as a reference the intensity 
at $408 \mathrm{~nm}$, associated with product formation (see Figure 1C). On the other hand, Figure $2 \mathrm{C}$ shows anticorrelated intensity fluctuations of reactant (at $538 \mathrm{~nm}$ ) and product (at $408 \mathrm{~nm}$ ) species over the course of the reaction. Figure 2D shows the correlation wavelength-dependent correlation coefficient averaged over the entire reaction time, thus confirming the assignment of spectral regions that are correlated, anticorrelated or non-correlated with the reactant.

Previously, we illustrated the utility of monitoring full spectra during a photochemical conjugated polymer degradation reaction, where the fluorescence lineshapes, in connection with ultrafast spectroscopy and multimode Brownian oscillator modelling, indicated time-evolving solute-solvent couplings.[12] In the present work, the results shown in Figure 1 illustrate the advantage of measuring time-dependent spectra for this complex reaction, since it shows chemical reaction interconversion between species, as indicated in Figures $1 \mathrm{C}$ and $2 \mathrm{C}$, and it also clearly shows the correlated time-dependent intensities during the chemical reaction (Figure 2), despite the complicated decays seen by looking at individual wavelengths (Figure 1A). We also note that application of the single-wavelength approach to the present reaction would not allow identification of the correlations shown in Figure 2. Since it is possible to perform single-wavelength kinetic measurements and time-dependent spectra with similar efficiency, we also highlight that each approach may be more suitable for specific applications. For example, in the case of homogeneous reactions with monotonic time-dependence of reactant and product concentrations, one usually chooses an individual wavelength to monitor reactant or product during the chemical kinetics measurement. On the other hand, for hydrogen peroxide disproportionation or other related reactions, the approach presented in Figure 1 may be a useful alternative.

\section{Time-dependent dynamic light scattering}

Figures 1 and 2 also show that, given the hydrogen peroxide disproportionation reaction studied in the present work, and depending on the reactant concentrations, oxygen bubbles can be visually observed (Movie S1) that also influence the kinetic measurements. To be more specific, the observed signal (Figure 1) during the chemical kinetics measurement studied here is a combination (sum) of absorbance and scattering. Therefore, we sought to study experimentally specific contributions to the observed kinetic response.

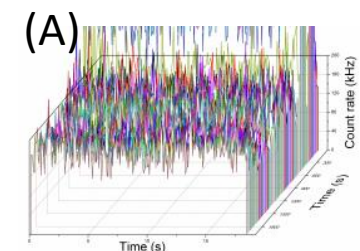

(F)

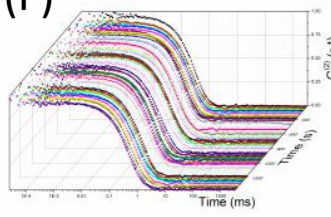

(B)

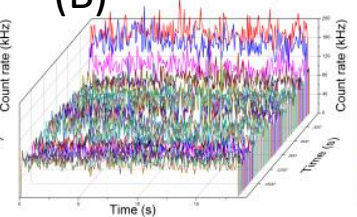

(G)

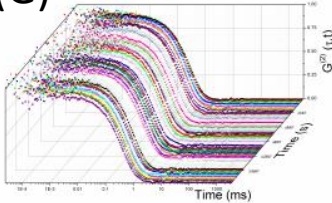

(C)

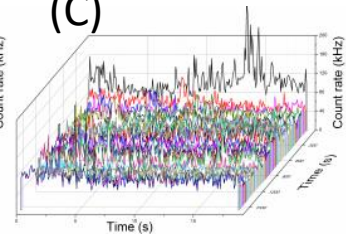

(H)

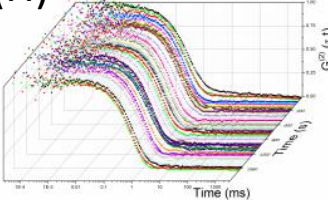

(D)

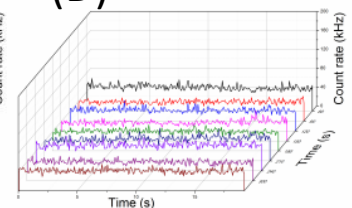

(I)

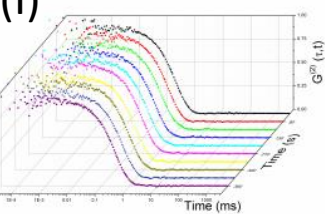

(E)

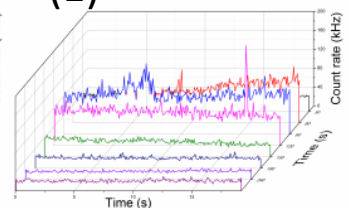

(J)

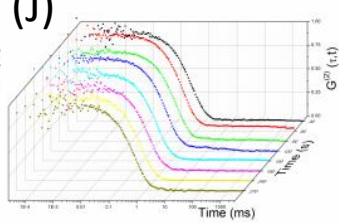

Figure 3. Time-dependent dynamic light scattering count rates (top) and associated time-dependent and time-correlation functions, $\mathrm{G}^{(2)}(\tau, \mathrm{t})$ (bottom) for the reaction between potassium permanganate and hydrogen peroxide at different initial concentrations (see text). 
In addition to the spectral characterization of permanganate reactant and manganese dioxide products (Figures 1 and 2 ) under conditions where macroscopic oxygen bubbles could be observed, we also studied the reaction in conditions (see Experimental Section) where macroscopic oxygen bubbles in bulk solution are minimized (although macroscopic bubbles can still be observed at the bottom of the container). Under these conditions, we studied light scattering and absorbance during the chemical reaction.[26-30] Thus, initially we performed a combined study of dynamic light scattering and chemical reaction kinetics. As outlined in Figure SI3, we used a multi-timescale perspective, where individual light scattering count rate traces, measured consecutively during the chemical reaction, were used to construct time-correlation functions in the nanosecond to millisecond timescale, followed by evaluation of timedependent time-correlation functions as the chemical reaction proceeds.

Figure 3 shows our results for dynamic light scattering $(D L S)$ measurements of the hydrogen peroxide disproportionation reaction between potassium permanganate and hydrogen peroxide in the concentration range shown in Table 1 where macroscopic oxygen bubbles are absent in solution. Thus, for each set of initial concentrations, Figure 2(top) shows the scattered light count rate traces (in $\mathrm{kHz}$ units) as a function of time during individual dynamic light scattering measurements, and as a function of reaction time (successive light scattering measurements). For each concentration, from each intensity trace shown in Figure 2(top), the corresponding time correlation functions as a function of correlation time (in the ns to $\mathrm{ms}$ range) obtained are shown in Figure 2(bottom), as well as successive time correlation functions as a function of chemical reaction time.

From the count rate traces, in comparing results obtained for different reaction conditions (Figures $3 \mathrm{~A}-3 \mathrm{E})$, we note that the same scale was used to show the intensities. Thus, Figures $3 \mathrm{~A}-3 \mathrm{E}$ indicate increasing overall scattered light intensity with increasing initial concentration, and that consecutive trace intensities vary during the chemical reaction. Throughout the experimental set shown, some of the traces also indicate transient events in which scattered light intensity increases. However, the individual count rate traces results exhibited a mean value and standard deviation following Gaussian statistics over the entire reaction course (see below). This observation confirms the dilute regime of single nanobubble scattering events in which the experiment is performed, also confirmed by retrieval of the corresponding time-correlation functions, as can be seen in Figures 3F-3J.
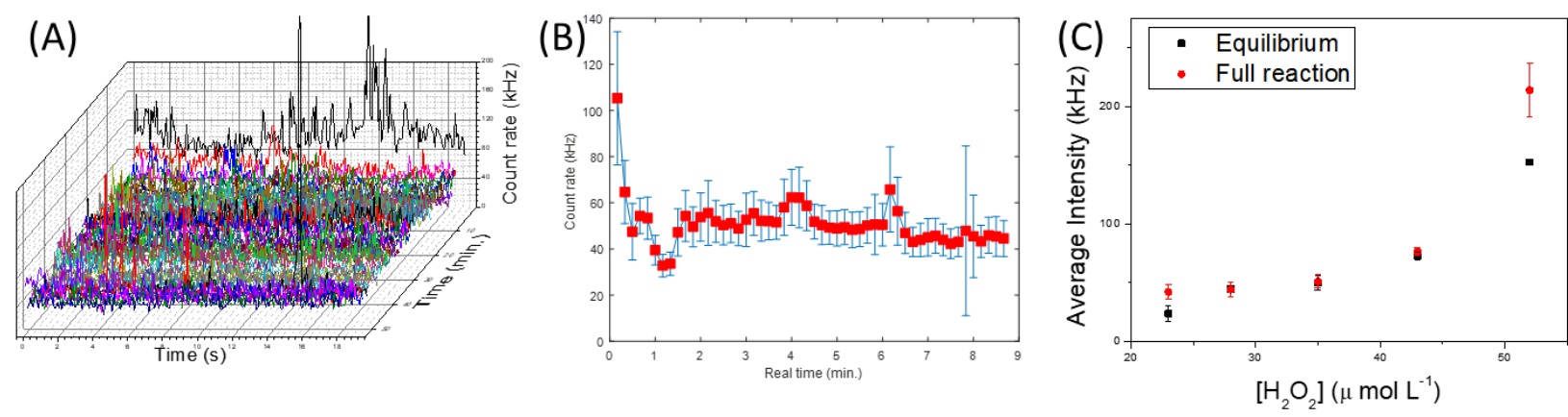

Figure 4. Analysis of time-dependent count rate traces as a function of reaction time. (A) Same as Figure 3C;

(B) Mean intensity (red squares) and standard deviation (blue) over the course of the chemical reaction; (C) Average count rate for the equilibrated system (black squares) and for the full reaction (red circles) as a function of concentration. 
Figure 4 shows the analysis of scattered light count rate traces as a function of time (for each trace) and over the course of the reaction (consecutive traces). This analysis was performed for the data set shown in Figures 3A-3E and is exemplified in Figures $4 \mathrm{~A}$ (same as Figure $3 \mathrm{C}$ ) and 4B. In Figure 4B, we show the mean count rate and corresponding standard deviation calculated for each individual trace shown in Figure 4A. Therefore, Figure 4B shows the trace-averaged time-evolution of scattered light over the course of the reaction, indicating larger fluctuations at early times and a nearly constant value (within experimental error) as the system reaches an equilibrated state. Even though absolute intensities from consecutive count rate traces varied of the course of each reaction, this reaction-averaged metric was useful for comparing reactions performed in different conditions. By repeating this analysis for the data set shown in Figures 3A-3E, we see in Figure $4 \mathrm{C}$ that the average intensity for both the full reaction (red) and at equilibrium (black) increases as a function of hydrogen peroxide initial concentration. Therefore, in the concentration range studied in this work, we see that the reaction-averaged intensity correlates with the initial hydrogen peroxide concentration, and thus with the amount of product formed.
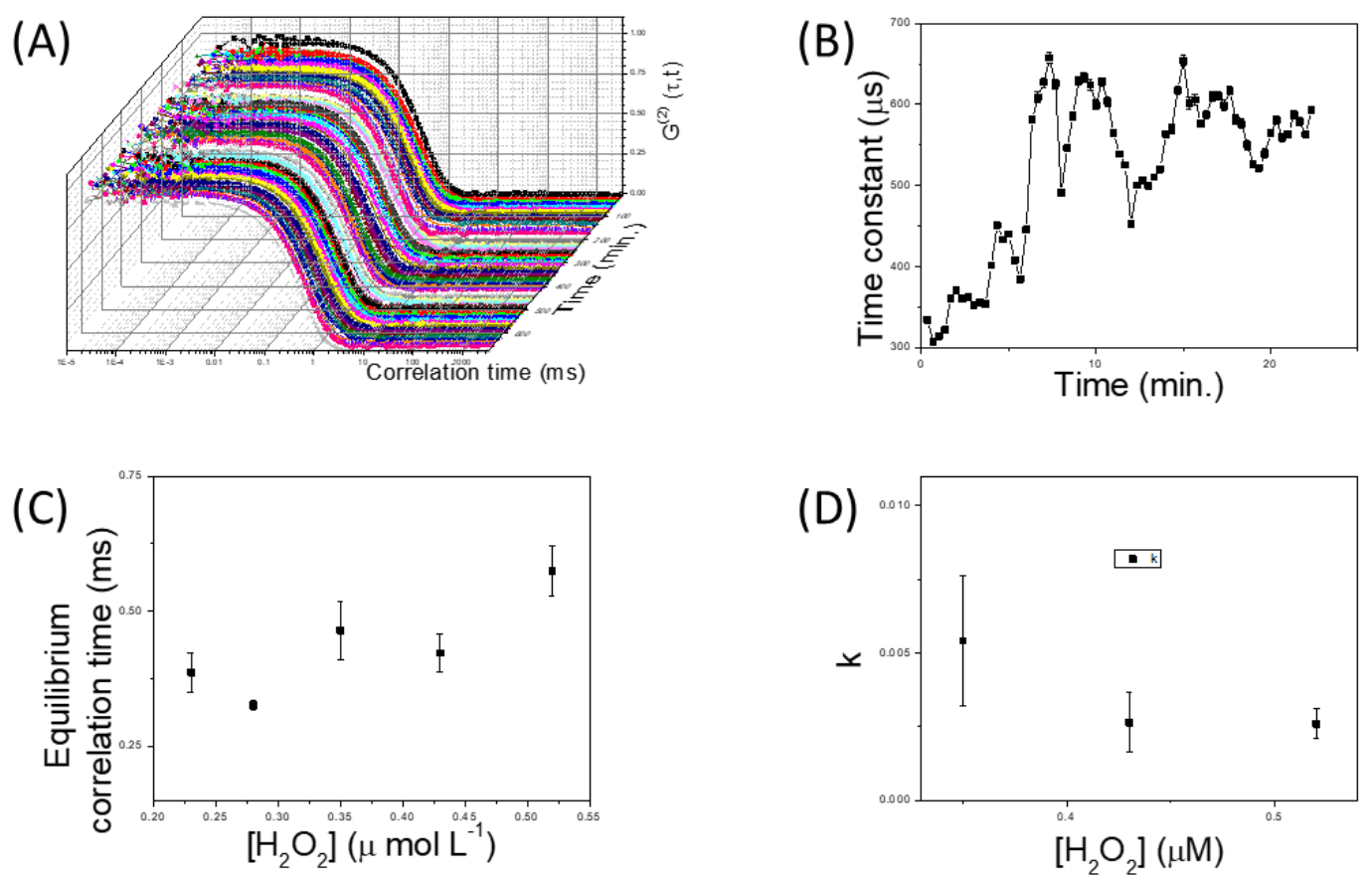

Figure 5. Analysis of time-correlation functions as a function of reaction time. (A) Same as Figure 3F; (B) correlation function decay time constants (blue) and amplitude (red) as a function of reaction time; (C) Equilibrium correlation time decay constant vs initial hydrogen peroxide concentration; (D) observed rate constant, kobs, vs initial hydrogen peroxide concentration.

Figure 5 shows analysis of the reaction time dependent time-correlation functions shown in Figures 3F-3J. Thus, Figure 5A shows consecutive correlation functions measured as the chemical reaction proceeded. Each correlation function was fitted to a single exponential decay function, and the resulting fitting parameters are shown in Figure $5 \mathrm{~B}$ with nearly normalized correlation function amplitudes and reaction-time-dependent correlation function decay constants. Figure $5 \mathrm{~B}$ shows a large variation of the DLS decay constants over the course of the reaction. Nonetheless, a net increase in the decay constants is observed at early times, and a plateau observed at longer times as the chemical reaction reaches equilibrium. By quantifying the equilibrium values from each set in Figures $3 \mathrm{~F}-3 \mathrm{~J}$, Figure $5 \mathrm{C}$ shows an approximately constant equilibrium correlation time, except at the highest initial hydrogen peroxide 
concentration studied in this work. Figure 5D shows that the observed reaction rate constant decreases with increasing concentration, although uncertainty increased at lower concentrations and at the lowest concentrations studied in this work we could not obtain adequate fits. Overall, we conclude that the results shown in Figure 5 indicate that the correlation function decay time constants increase over the course of the reaction, reaching an equilibrium value that is independent of initial hydrogen peroxide concentration, except at the highest concentration studied in this work.

We also note that the correlation function decay constants are associated with the hydrodynamic radius of the corresponding Brownian particle, which in the present case is an oxygen nanobubble.[26-30] From the data shown in Figures 2-4, we can thus further connect the equilibrium-averaged correlation times and count rates to nanobubble size and concentration, respectively. The dynamic light scattering measurements at the range of concentrations indicated in Table 1 showed that, under appropriate experimental conditions (initial reactant concentrations, overall reaction time, and colloidal system size) as shown here, it is possible to monitor oxygen nanobubble product formation by way of scattering. The results shown in Figures 3-5 illustrate the utility of measuring the full response (scattering and absorption) during the chemical reaction. Under the conditions shown above, which lead to stable nanobubbles and negligible amount of larger bubbles in solution, we emphasize that light scattering occurs from individual particles traversing the path of incident beam of light in the sample holder, and thus correspond to the low intensity, dilute regime. Thus, we can perform chemical kinetics measurements following reagents and products at $458 \mathrm{~nm}$ and $400 \mathrm{~nm}$, respectively, and connect the corresponding extinction measurements with absorbance. Figure SI shows the resulting chemical kinetics profiles and the corresponding decay and formation constants for reactants and products, respectively. Even under these conditions optimized for the observation of nanobubble formation and thus small light scattering contributions to the observed spectroscopic signal, we observed large absorbance fluctuations, and we note a net absorbance change throughout the measurement time, which was much longer than the reaction times inferred from Figures 3-5. Thus, the absorbance measurements indicated additional contributions to the observed spectroscopic signal during our study of hydrogen peroxide disproportionation.

\section{Chemical kinetics and Information theory}

Due to the nanoscale bubble size and the associated refractive index, visual inspection of the solutions over the course of the reaction did not show evidence of bubbles in solution, as expected. Connecting with the absorbance measurements and within the context of a chemical kinetics study of reaction mechanisms using uv-vis spectrophotometers, the signal measured corresponds to light intensity transmitted through the sample. Therefore, for the present reaction and concentration range, the signal measured with a uv-vis spectrophotometer accounts for extinction, which is the sum of absorption and scattering. In addition to the uv-vis absorbance and dynamic light scattering kinetics studies described above, we also note that visual inspection of the reaction indicated that several processes, concurrent with the chemical reaction per se, occur over the course of the reaction time: diffusion of reactants and products, convection, interaction of macroscopic bubbles with the bottom of the cuvette, and coupled reaction-transport processes. These observations help understand the noisy data obtained from the spectroscopic measurements. On the other hand, a quantitative assessment of such observations was performed to shed additional light to these processes.

To achieve this goal, we also monitor the same reactions by looking at the entire sample with a camera, perform quantitative imaging and connect with spectroscopy.[12] First, by visual inspection of the resulting recorded movies, a multitude of reaction-transport processes can be identified (see Supporting 
Information). Therefore, the chemical reaction and its spectroscopic monitoring by uv-vis spectroscopy must be treated with a different approach than that normally used in the study of homogeneous, wellmixed reactions. Considering the light-matter interaction processes that occur during a kinetics measurement, a beam of light traverses the sample in a specific, small portion of the sample. Given that this reaction under study is heterogeneous as described above, probing a small portion of the entire system leads to a study of that sample region, rather than a study of the chemical reaction per se. This observation helps understand the previous measurements of absorbance kinetics as well as dynamic light scattering. Quantitative analysis of this reaction was performed to better understand the contributions of reactions and transport to the observed time-dependent absorbance changes.

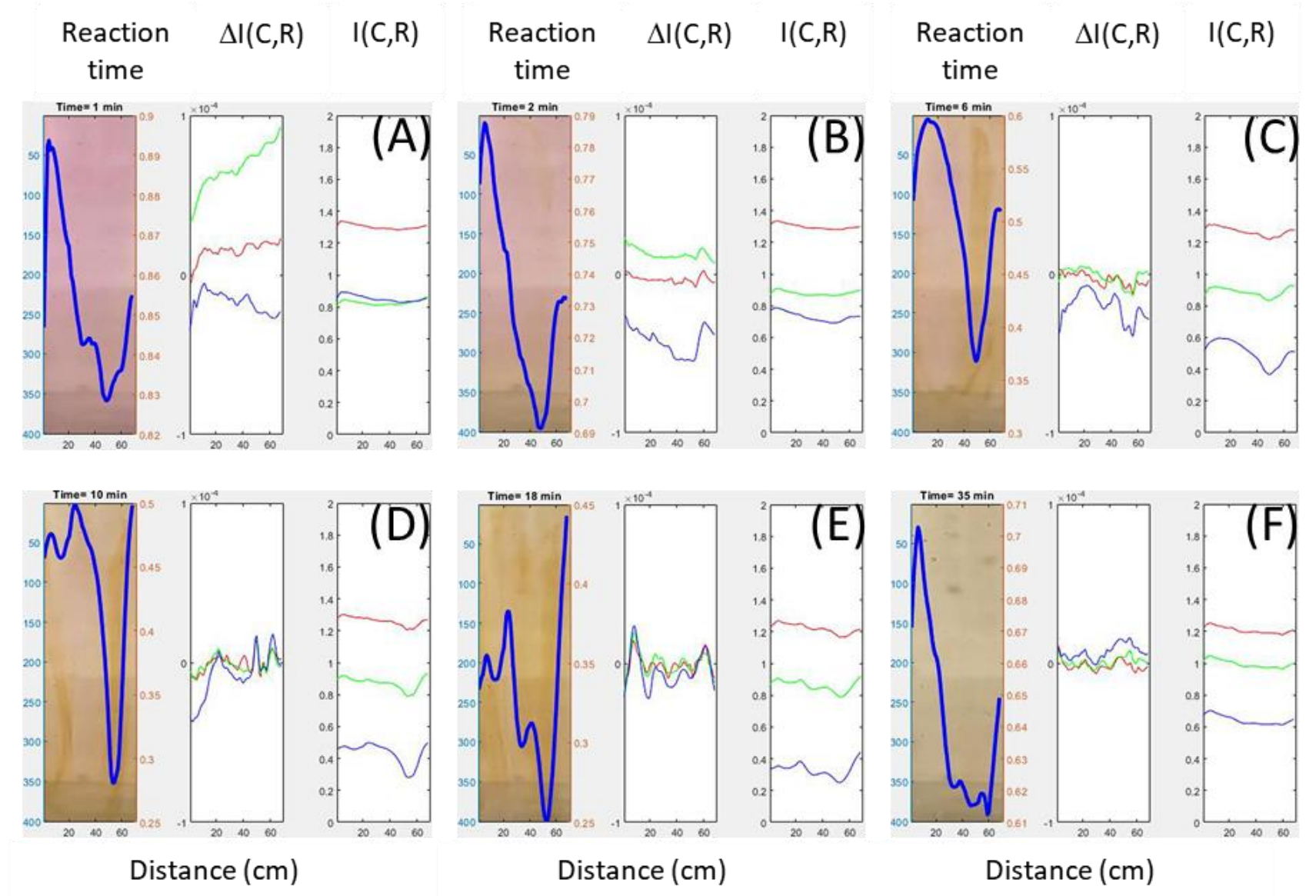

Figure 6. Chemical reaction time-dependent mutual information from LUB algorithm using vertical partitioning. For each figure, on the left is shown an image of the reaction overlaid with the (rescaled) mutual information for the case of $B$ color and $V$ (vertical partitioning); the center plot shows mutual information difference, $\Delta l(C, R)$, and the right plot shows the mutual information $I(C, R)$.

Movie S2 shows chemical reaction monitoring overlaid with the mutual information $I(C, R)$ calculated using vertical image partitioning and blue color, which corresponds to product formation. Since the quantity $I(C, R)$ changes in magnitude as a function of vertical partitioning location as well as reaction time, in Movie S2 I(C,R) was rescaled to show more clearly the connection between chemical reaction and information theory. While Movie S2 shows a sequence of images taken at $30 \mathrm{fps}$ over the course of the reaction, Figure 6 shows results of this analysis performed at individual frames (reaction times): (A) 1 min.; (B) 2 min.; (C) 6 min.; (D) 10 min.; (E) 18 min.; and (F) 35 min. For each frame, we show three results: (1) an image of the reaction at the time indicated overlaid with the rescaled $I(C, R)$ obtained from blue color and 
vertical partitioning across the sample (left); (2) the difference mutual information $\Delta l(C, R)$ across the red, green and blue palette (center); and $(3)$ the mutual information $I(C, R)$ magnitude across red, green and blue.

In comparing information gain during the chemical reaction, the quantitative analysis in Figure 6 shows that vertical partitioning leads to larger net magnitude changes in mutual information $I(C, R)$ for the blue color, assigned to the product. On the other hand, in the case of mutual information difference, $\Delta \mathrm{I}(\mathrm{C}, \mathrm{R})$, larger net magnitude changes are observed for green color, which we assigned to the reactant. During the measurement time, we thus find a correspondence between $I(C, R)$ and product diffusion occurring over the entire reaction time; on the other hand, $\Delta I(C, R)$ and reactant consumption changes are observed only at early times. Although the timescale separation between chemical reaction and product diffusion can, in principle, be inferred from MovieS2 and Figure 6, we should mention that there is no sharp distinction or boundary between chemical reaction occurring at early times and product diffusion occurring at later times. Rather, $\Delta l(C, R)$ shows negligible fluctuations over time after $\sim 5$ minutes for the entire color palette. Overall, quantitative analysis using vertical partitioning by the LUB algorithm (Figure 6) allows identification of different processes occurring during video-rate imaging monitoring of the reaction between potassium permanganate and hydrogen peroxide.

We have also studied horizontal partitioning of each frame during the chemical reaction, and calculated $\mathrm{I}(\mathrm{C}, \mathrm{R})$ and $\Delta \mathrm{I}(\mathrm{C}, \mathrm{R})$ for the RGB color palette (Figure S5). As shown in Figure S2, the magnitude of $I(C, R)$ is over an order of magnitude smaller than the corresponding magnitude calculated using vertical partitioning. In comparing horizontal partitioning $I(C, R)$ magnitudes over the course of the reaction, we note that horizontal partitioning finds correspondence with reactant concentration changes over time. Therefore, from I $(C, R)$, the chemical reaction occurs during the first few minutes, same conclusion reached in the analysis based on vertical partitioning and dynamic light scattering. Moreover, I(C,R) also shows the reaction is more pronounced near the bottom of the sample holder, an information that cannot be inferred from analysis based on vertical partitioning. Therefore, from the horizontal partitioning, both visual inspection and $\mathrm{I}(\mathrm{C}, \mathrm{R})$ quantitative analysis indicate that the product formation occurs during the first few minutes.

Within the goals of this research project, the results shown in Figures 1-6 indicate that the chemical reaction chosen as a model system presents the challenges we expected to address. Specifically, the chemical reaction involves small-molecule reactants and products, while also involving bubble and colloid particle / aggregate formation, besides transport professes, including diffusion and drift. These are aspects that are expected to appear in the study of other reactions important for energy- and materials-related fields.

\section{Coupled stochastic and macroscopic reaction-diffusion numerical simulations}

Figures $7 A$ and $7 B$ show the temporal and spatial dependence of reactant $A$ over the course of the reaction $A+B \rightarrow C$. The initial concentrations of $A$ and $B$ are the same but their spatial distribution differs to mimic more heterogeneous reaction conditions. Specifically, the species $A$ is uniformly distributed over the entire simulation region, while $B$ is located at a specific small portion of the total simulation region, as can be inferred from Figure 7A at early times. Figure 7A shows the spatio-temporal simulation results whereas Figure $6 \mathrm{~B}$ is used to compare kinetics at individual locations with the spatially-averaged kinetics and theory. The stochastic simulation parameters are the same described in our previous work. Thus, for the specific values of diffusion coefficient and forward reaction rate constant, Figure 7B shows a good agreement 
between the spatially-averaged decay and the analytical result for this reaction, even though the individual temporal profiles differ markedly from the average. In connecting with our spectroscopy probe of a complex, heterogeneous reaction, we see from Figures $7 \mathrm{~A}$ and $7 \mathrm{~B}$ that different apparent reaction rate constants may be extracted from the simulation results depending on the sample region used to perform the analysis. Moreover, by considering the entire simulation region, the correct reaction rate constant can be recovered.
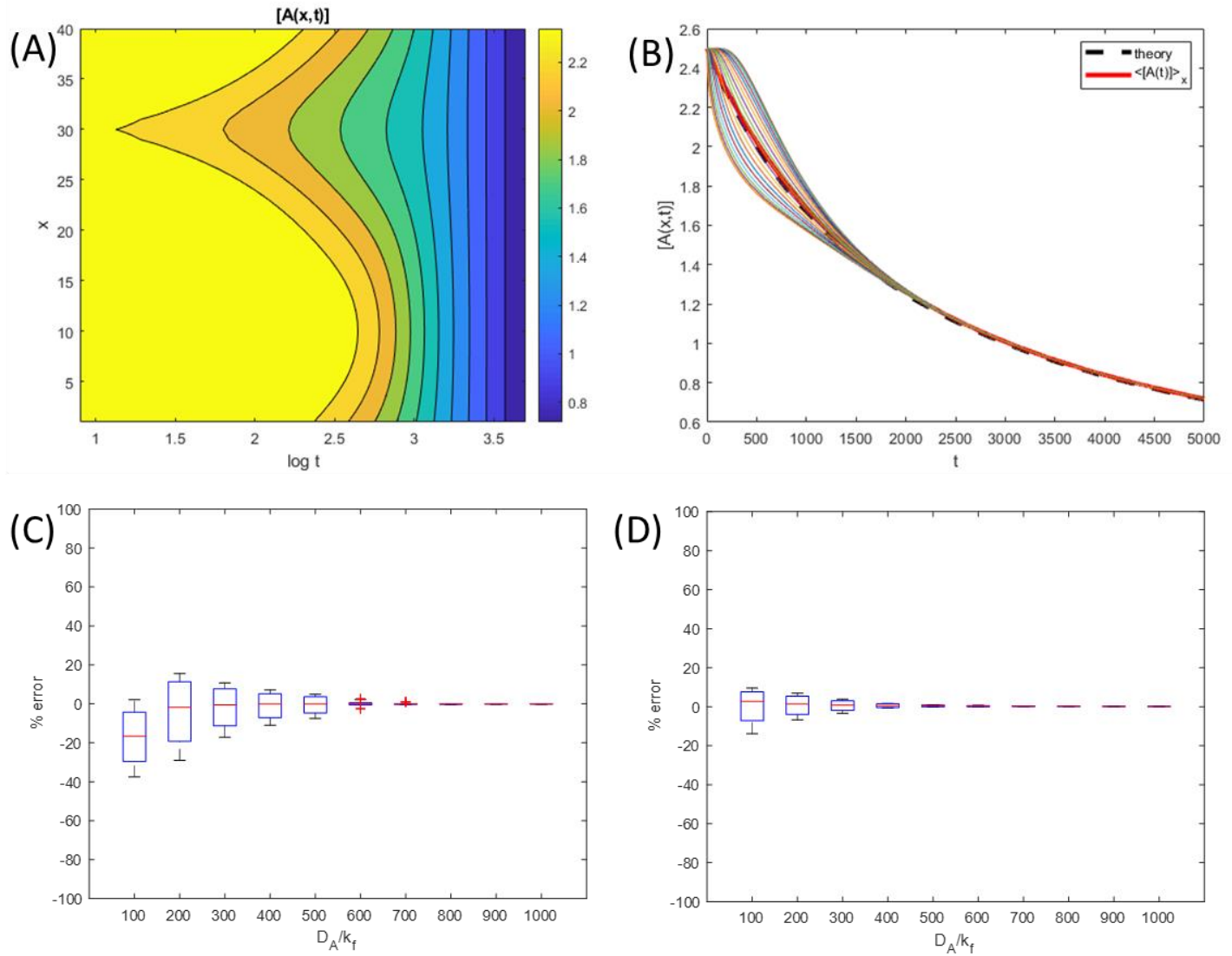

Figure 7. Coupled analysis of reaction-diffusion system at the macroscopic and microscopic level and its connection to spectroscopy: $A+B \rightarrow C$. (A) Temporal and spatial dependence of reactant concentration, $[A]$. (B) Time-dependent variation of $[A]$ across space (thin colored curves), spatially average $[A(t)]$ (thick red curve) and analytical solution (thick dashed black curve); (C) relative error in numerical result as a function of reactant $A$ diffusion coefficient scaled by forward reaction rate constant, with $D A=D B$; (D) same as (C) but with $D B=5 * D A$.

The results shown in Figures 7A and 7B were obtained for a specific set of parameters such that DA $=\mathrm{DB}$ and $\mathrm{DA} / \mathrm{kf}=500$. By comparing the relative error of the numerical result and the analytical result, we also studied the effects of varying $\mathrm{DA} / \mathrm{kf}$ at equal reactant diffusion coefficient and with $\mathrm{DB}=5^{*} \mathrm{DA}$, as shown in the boxplots in Figures $7 C$ and 7D. In both cases, we see that, for sufficiently large DA/kf, the difference between numerically retrieved and analytical result is negligible, and that the same result is obtained regardless of whether we analyze a specific sample region or the entire sample. This regime is relevant for many spatially homogeneous and/or well-stirred chemical reactions, as mentioned previously. On the other hand, for both case studies shown, as the ratio DA/kf decreases, the error increases, and it is necessary to monitor the entire observation region. This regime is relevant for spatially heterogeneous reactions, systems that cannot be stirred, or under conditions where larger particles are present. In comparing Figures $7 C$ and $7 D$, we note that, at low $D A / k f$ ratio, the mean numerically retrieved rate 
constant differs from the correct value when $D A=D B$ but not when $D B=5 * D A$, further indicating conditions for which monitoring the entire sample gives correct results, even for spatially heterogeneous systems.

In conclusion, we report a spectroscopic study of hydrogen peroxide disproportionation by potassium permanganate. Adding to previous work on the same reaction, we measure spectra, absorbance and scattering, imaging, complementing the measurements with analysis and simulations. Compared with single-wavelength monitoring, measuring the entire spectrum over time enabled a correlation analysis of reactants, products, and scattering, thereby contributing to spectroscopic assignment to each reacting species, even in reactant concentration conditions where the reaction exhibited macroscopic bubbles and transport processes. Monitoring the full linear optical response (absorbance and scattering) in experimental conditions where macroscopic bubbles were absent in bulk solution enabled the study of oxygen nanobubble product formation kinetics and equilibrium. By monitoring transmitted light through the entire sample holder, together with information-theoretic analysis, we characterized different contributions (mixing, chemical reaction, transport and diffusion) to the observed signal. Simulations combining microscopic (stochastic) and macroscopic descriptions of chemical reaction and diffusion illustrated the differences in information content obtained when monitoring the entire system vs a subset of the simulation region. This reaction is well-known, and it exhibits many features (spatial and dynamical heterogeneities, bubble and aggregate formation and growth, transport effects etc) that are observed in other reactions of much current interest, such as solar fuels, artificial photosynthesis, and renewable energy reactions. The reaction which is the focus of the present work is difficult to study using tools of chemical kinetics developed for the study of homogeneous systems. Thus, overall, the results presented here exemplify a set of experimental and numerical tools that may help understanding chemical reactions in heterogeneous conditions.

\section{ACKNOWLEDGEMENTS}

We thank the financial support from FAPESP (the Sao Paulo Research Foundation) for grant 2017/11986-5, CNPQ (the National Council for Scientific and Technological Development) INCT Catalysis in Molecular and Nanostructured Systems and CAPES for a graduate fellowship. We also thank Shell and the strategic importance of the support given by ANP (Brazil's National Oil, Natural Gas and Biofuels Agency) through the R\&D levy regulation. We thank Guilherme Oliveira for his help with the imaging software usage.

\section{BIBLIOGRAPHY}

1. K. Kohse-Hoinghaus, J. Troe, J.-U. Grabow, M. Olzmann, G. Friedrichs K.-D. Hungenberg, Phys. Chem. Chem. Phys. 2018, 20, 10561.

2. S.J. Klippenstein, V.S. Pande, D.G. Truhlar, J. Am. Chem. Soc. 2014, 136, 528-546.

3. Z. Huang R. Boulatov, Chem. Soc. Rev. 2011, 40, 2359-2384.

4. J. Ross, M.O. Vlad, Annu. Rev. Phys. Chem. 1999, 50, 51-78.

5. B. Peters, Annu. Rev. Phys. Chem. 2016, 67, 669-690.

6. S. van Bavel, S. Verma, E. Negro, M. Bracht, ACS Energy Lett. 2020, 5, 2597-2601.

7. A. Nagar T. Pradeep, ACS Nano 2020, 14, 6420-6435.

8. C.J. Miller, A.L. Rose, T.D. Waite, Front. Mar. Sci. 2016, 3,134.

9. Y. Shiraishi, M. Matsumoto, S. Ichikawa, S. Tanaka, T. Hirai, J. Am. Chem. Soc. 2021, 143, 12590-12599. 
10. J.C. Franco, G. Gonçalves, M.S. Souza, S.B.C. Rosa, L.M. Thiegue, T.D.Z. Atvars, P.T.V Rosa, R.A. Nome, Opt. Exp. 2013, 21 (25), 30874-30885.

11. P.T.C. Loiola, W.D. Dias, M.S. Souza, G.F. Ferbonink, M.G. Zaniolo, M.F. Cerini, T.D.Z. Atvars, J. Phys. Org. Chem. 2014, 27 (4), 316-321.

12. R.A. Nome, A.F. Costa, J. Lepkoski, G.A. Monteiro, J.G. Hayashi, C.M.B. Cordeiro, ACS Omega 2017, 2 (6), 2719-2727.

13. G.H. Oliveira, M.T. Galante, T.T. Martins, L.F.L.S. dos Santos, F. Ely, C. Longo, R.V. Gonçalves, S.R. Muniz, R.A. Nome, Solar Energy 2019, 190, 239-245.

14. R.H. Simoyi, P. de Kepper, I.R. Epstein, K. Kustin, Inorg. Chem. 1986, 25, 538-542.

15. A. Nagy, L. Treindl, J. Phys. Chem. 1989, 93, 2807-2810.

16. O.F. Steinbach, J. Chem. Educ. 1944, 21, 66.

17. J. Ross, J. Phys. Chem. A 2008, 112, 2134-2143.

18. I. S. Shin, S. K. Han, K. M. Lee, S. B. Lee, H. W. Jung, New Phys. Sae Mulli 2013, 63, 655-660.

19. B. Lee, M.K. Seob, D. Kim, I.-S. Shin , M. Schich, H. Jeong, S.K. Han, PNAS 2020, 117(43), 2658026590.

20. D.N. Reshef, Y.A. Reshef, H.K. Finucane, S.R. Grossman, G. McVean, P.J. Turnbaugh, E.S. Lander, M. Mitzenmacher, P.C. Sabeti, Science 2011, 334 (6062), 1518-1524.

21. V. Méndez, S. Fedotov, W. Horsthemke, Reaction-Transport Systems, Springer Series in Synergetics, Heidelberg, 2010.

22. D.T. Gillespie, J. Phys. Chem. 1977, 81, 2340-2361.

23. E. Roberts, J.E. Stone, Z. Luthey-Schulten, J. Comput. Chem. 2013, 34(3), 245-255.

24. G. Volpe, S. Gigan, G. Volpe, Am. J. Phys. 2014, 82, 659-664.

25. R.A. Nome, C. Sorbello, M. Jobbágy, B.C. Barja, V. Sanches, J.S. Cruz, V.F. Aguiar, Meth. Appl. Fluor. 2017, 5 (1), 014005.

26. G.S. Manning, Phys. Chem. Chem. Phys. 2020,22, 17523-17531.

27. P. Bhandari, X. Wang, J. Irudayaraj, ACS Nano 2017, 11, 2682-2688.

28. Y. Ji, Z. Guo, T. Tan, Y. Wang, L. Zhang, J. Hu, Y. Zhang, ACS Omega 20216 (4), 2873-2881.

29. T. Vehmas, L. Makkonen, ACS Omega 2021, 6, 8021-8027.

30. F.Y. Ushikuboa, T. Furukawaa, R. Nakagawaa, M. Enaria, Y. Makinoa, Y. Kawagoea, T. Shiinab, S. Oshita, Coll. Surf. A: Phys. Eng. Asp. 2010, 361(1-3), 31-37. 